\title{
A representative reference for MRI-based human axon radius assessment using light microscopy
}

\author{
Laurin Mordhorst ${ }^{\mathrm{a}, *}$, Maria Morozova ${ }^{\mathrm{b}, \mathrm{c}}$, Sebastian Papazoglou ${ }^{\mathrm{a}}$, Björn Fricke ${ }^{\mathrm{a}}$, Jan \\ Malte Oeschger ${ }^{\mathrm{a}}$, Thibault Tabarin ${ }^{\mathrm{a}}$, Henriette Rusch ${ }^{\mathrm{c}}$, Carsten Jäger ${ }^{\mathrm{b}}$, Stefan Geyer ${ }^{\mathrm{b}}$, \\ Nikolaus Weiskopf ${ }^{\mathrm{b}, \mathrm{d}}$, Markus Morawski ${ }^{\mathrm{b}, \mathrm{c}}$, Siawoosh Mohammadi ${ }^{\mathrm{a}, \mathrm{b}}$ \\ ${ }^{a}$ Institute of Systems Neuroscience, University Medical Center Hamburg-Eppendorf, Hamburg, \\ Germany \\ ${ }^{b}$ Department of Neurophysics, Max Planck Institute for Human Cognitive and Brain Sciences, Leipzig, \\ Germany \\ ${ }^{c}$ Paul Flechsig Institute of Brain Research, Medical Faculty, Leipzig University, Leipzig, Germany \\ ${ }^{d}$ Felix Bloch Institute for Solid State Physics, Leipzig University, Leipzig, Germany
}

\begin{abstract}
Non-invasive assessment of axon radii via MRI bears great potential for clinical and neuroscience research as it is a main determinant of the neuronal conduction velocity. However, there is a lack of representative histological reference data on the scale of the cross-section of MRI voxels for validating the MRI-visible, effective radius $\left(r_{\text {eff }}\right)$. Because the current gold standard stems from neuroanatomical studies designed to estimate the frequency-weighted arithmetic mean radius $\left(r_{\text {arith }}\right)$ on small ensembles of axons, it is unsuited to estimate the tail-weighted $r_{\text {eff }}$. We propose CNN-based segmentation on high-resolution, large-scale light microscopy (lsLM) data to generate a representative reference for $r_{\text {eff. }}$ In a human corpus callosum, we assessed estimation accuracy and bias of $r_{\text {arith }}$ and $r_{\text {eff. }}$. Furthermore, we investigated whether mapping anatomy-related variation of $r_{\text {arith }}$ and $r_{\text {eff }}$ is confounded by low-frequency variation of the image intensity, e.g., due to staining heterogeneity. Finally, we analyzed the potential error due to outstandingly large axons in $r_{\text {eff }}$. Compared to $r_{\text {arith }}, r_{\text {eff }}$ was estimated with higher accuracy (normalized-root-mean-square-error of $r_{\text {eff: }}: 7.2 \% ; r_{\text {arith }}: 21.5 \%$ ) and lower bias (normalized-mean-bias-error of $r_{\text {eff }}:-1.7 \% ; r_{\text {arith }}: 16 \%$ ). While $r_{\text {arith }}$ was confounded by variation of the image intensity, variation of $r_{\text {eff }}$ seemed anatomy-related. The largest axons contributed between $0.9 \%$ and $3 \%$ to $r_{\text {eff }}$. In conclusion, the proposed method accurately estimates $r_{\text {eff }}$ at MRI voxel resolution across a human corpus callosum sample. Further investigations are required to assess generalization to brain areas with different axon radii ensembles.
\end{abstract}

Keywords: Deep learning, Histological MRI, Cross microscopy, Neuroanatomy, Axon radii distribution

*Corresponding author 


\section{List of Symbols and Acronyms}

\section{Symbols}

\section{Axon radii ensemble}

$\begin{array}{ll}\mathrm{r} & \text { individual axon radius } \\ \boldsymbol{r} & \text { axon radii ensemble } \\ \tilde{\boldsymbol{r}} & \text { reference axon radii ensemble } \\ \tilde{\boldsymbol{r}}_{\mathrm{EM}} & \text { EM-based axon radii ensemble obtained through anno- } \\ & \text { tation of } S_{\mathrm{EM}} \\ \tilde{\boldsymbol{r}}_{\text {lsLM,large }} & \text { lsLM-based axon radii ensemble for large axons obtained } \\ & \text { through annotation of } S_{\text {lsLM,large }} \\ \hat{\boldsymbol{r}} & \text { estimated axon radii ensemble } \\ \hat{\boldsymbol{r}}_{r n g} & \text { estimated axon radii ensemble with erroneous radii in } \\ & \text { axon radii range } r n g\end{array}$

\section{Axon radii ranges}

\section{small $\quad$ axons with $r<0.3 \mu \mathrm{m}$}

medium-sized axons with $0.3 \mu \mathrm{m} \leq r<1.8 \mu \mathrm{m}$

large $\quad$ axons with $r \geq 1.8 \mu \mathrm{m}$

\section{Arithmetic mean axon radius}

$r_{\text {arith }}$
$\tilde{r}_{\text {arith }}$
$\hat{r}_{\text {arith }}$

arithmetic mean radius

reference arithmetic mean radius

estimated arithmetic mean radius

\section{MRI-visible, effective axon radius}

$r_{\text {eff }}$
$\tilde{r}_{\text {eff }}$
$\hat{r}_{\text {eff }}$
$\hat{r}_{\text {eff, } r n g}$

effective radius

reference effective radius

estimated effective radius

estimated effective radius based on an axon radii ensemble with erroneous radii in axon radii range $r n g$

\section{Subsections}

$\begin{array}{ll}S_{\mathrm{EM}} & \text { EM subsection } \\ S_{\text {lsLM }} & \text { small lsLM subsection } \\ S_{\text {lsLM,large }} & \text { large lsLM subsection }\end{array}$




\section{Acronyms \\ CNN convolutional neural network}

EM electron microscopy

lsLM high-resoution, large-scale light microscopy

NMBE normalized-mean-bias-error

NRMSE normalized-root-mean-square-error

\section{Introduction}

The MRI signal generated by an ensemble of protons probing the local, microscopic environment in human brain tissue contains information about microstructural tissue features such as the axonal radius [1, 2, 3, 4, The axonal radius is a key to determine neuronal communication in the human brain because it is related to, e.g., the neuronal conduction velocity [5, 6, 7]. The estimation of the axonal radius and other microstructural features via biophysical modeling of the MRI signal 8 is an active area of research because of its potential to partially replace or complement invasive ex-vivo histology with non-invasive, in-vivo histological MRI (hMRI) [9, 10] approaches. However, before these models can be used, they need to be validated against a robust histological reference. 11 .

The validation for the MRI-visible, effective radius $\left(r_{\text {eff }}\right)$ is currently lacking a robust, histological reference for human brain tissue. Since $r_{\text {eff }}$ is indicative of large, sparsely occurring axons, i.e., the tail of the axon radii distribution [1, 12, 13, it is essential to accurately capture the tail of the axon radii distribution in large samples to representatively estimate $r_{\text {eff }}$ for MRI voxels of a human MRI system ( $1 \mathrm{~mm}^{3}$ or larger). For perfusion-fixed rats, validation of $r_{\text {eff }}$ has been attempted on histological images of the cross-sectional size of ultra-high-resolution MRI voxels $\left(\sim 100 \mathrm{~mm}^{3}\right)$ using a preclinical MRI system [1]. However, validation on human brain tissue remains of interest, because it is unclear whether the validation of MRI-based models on rats can be translated to the human brain. As the tail of the axon radii distribution may vary between humans and other mammals 14, 15, $r_{\text {eff }}$ for humans may be shifted with respect to other species. This shift may be further reinforced by the reduced capability to resolve small axons in human MRI systems when compared to preclinical MRI systems 1, 16. For human brain, the current gold standard for the validation of $r_{\text {eff }}$ [1, 2, 21, 22] stems from neuroanatomical studies [17, 18, 19, 20] of small ensembles of axons (100-1000 axons), aiming to evaluate the arithmetic mean radius $\left(r_{\text {arith }}\right)$ on manually annotated electron microscopy images (EM). As $r_{\text {arith }}$ is weighted towards the bulk of axon radii, it can be expected that estimates of $r_{\text {arith }}$ are less sensitive to the ensemble size as compared to $r_{\text {eff }}$. For $r_{\text {eff }}$, however, small-ensemble estimates can strongly under- or overestimate $r_{\text {eff }}$ 23. of typical MRI voxels, because the tail of the axon radii distribution is insufficiently sampled.

Albeit high-resolution, large-scale light microscopy (lsLM) cannot resolve small axons as accurately as EM, an lsLM-based approach might be appropriate to generate a 
histological gold standard for the validation of MRI-based radius estimation in human brain tissue. Because of its large field-of-view, covering cross-sections of $1 \mathrm{~mm}^{2}$ or larger, lsLM enables the generation of large ensembles of axons including $10^{5}$ to $10^{6}$ axons per section and thus allows for more accurate sampling of the tail of the axon radii distribution. Moreover, lsLM has the advantage of being fast, cheap and simple to perform. As the assessment of axon radii on large field-of-view microscopy data renders manual annotation infeasible, automated approaches, e.g., methods based on convolutional neural networks $(\mathrm{CNN})$, are required. So far, CNN-based methods based on large two- or three dimensional scanning or transmission electron microscopy (SEM/TEM) sections have been trained on images of perfusion-fixed mice or rats 24, 25]. However, it is unlikely that the models generated in these studies translate well to immersion-fixed human brain tissue with higher tissue degradation.

In this study, we investigate the potential of lsLM and CNN-based segmentation to map the distribution of axon radii in a human corpus callosum specimen. We quantify the capability of the proposed method to estimate the MRI-visible $r_{\text {eff }}$ and the $r_{\text {arith }}$, which is commonly reported in neuroanatomical studies, by evaluating the estimation errors on six lsLM sections. While reference data for the frequency-weighted $r_{\text {arith }}$ can be generated through manual annotation with reasonable effort, the tail-weighting of $r_{\text {eff }}$ introduces the necessity to accurately capture the tail of the axon radii distribution and thus investigate larger ensembles of axons than can be realistically annotated. To address this challenge, we follow a three-fold approach to assess the contribution of different axon radii ranges towards $r_{\text {eff. }}$ First, the error due to badly-resolved, small axons is assessed in a cross-microscopy comparison using annotated EM sections from close spatial proximity as a reference. Second, the error due to medium-sized axons, that represent the bulk of the axon ensemble, is assessed using Monte Carlo methods. Third, the error due to large axons, is assessed based on exhaustive annotation of large axons in large field-ofview sections. Additionally, we investigate whether our method is capable of capturing anatomy-related, spatial variation of $r_{\text {arith }}$ and $r_{\text {eff }}$ in the presence of low-frequency image intensity variation, e.g., due to staining heterogeneity. Finally, we analyze the potential error due to individual, outstandingly large axons in $r_{\text {eff. }}$.

\section{Materials and methods}

\subsection{Ensemble mean axon radii}

In the following, we define an axon radii ensemble of $B$ individual radii $r_{b}$ with $b \in[1, B]$ as

$$
\boldsymbol{r}=\left(r_{1}, \ldots, r_{B}\right) \top .
$$

After binning axon radii of this ensemble into $K$ bins, the arithmetic mean radius can be defined as

$$
r_{\text {arith }}=\sum_{k=1}^{K} w_{\text {arith }, k} \cdot r_{k} \quad \text { with } \quad w_{\text {arith }, k}=\frac{n_{k}}{B},
$$


Table 1: The dataset of human tissue samples. The following tissue samples were investigated: a corpus callosum (CC), a corticospinal tract (CST) an optic chiasm (OC) and an anterolateral system (AS). Sections were assigned exclusively to the training or test dataset.

\begin{tabular}{rlcrrr}
\hline & & & & \multicolumn{2}{c}{ Sections } \\
\cline { 3 - 5 } Sample & Type & Size $\left[\mathrm{mm}^{2}\right]$ & Total & Training & 1 \\
OC & lsLM & 2.49 & 1 & 1 & Test \\
CST & lsLM & 12.71 & 1 & 1 & 6 \\
AS & lsLM & 4.86 & 10 & 6 & 6 \\
CC & lsLM & 0.34 to 9.16 & 6 & \\
CC & EM & 0.01 & &
\end{tabular}

where $n_{k}$ is the number of axons with radius $r_{k}$. Analogously, the MRI-visible, effective mean radius [1, 12, 13, can be defined as

$$
r_{\mathrm{eff}}=\sqrt[4]{\sum_{k=1}^{K} w_{\mathrm{eff}, k} \cdot r_{k}} \quad \text { with } \quad w_{\mathrm{eff}, k}=\frac{n_{k}}{B} \cdot \frac{r_{k}^{5}}{\frac{1}{B} \sum_{k=1}^{K} r_{k}^{2}}
$$

While $r_{\text {arith }}$ is frequency-weighted $\left(w_{\text {arith }, k}\right), r_{\text {eff }}$ is weighted $\left(w_{\text {eff, } k}\right)$ towards the tail of the axon radii distribution because $w_{\text {eff, } k}$ scales with the fifth power of $r_{k}$.

In the following, we denote vectors as bold-faced, estimations with a hat and reference values with a tilde. Thus, we denote estimations of individual radii, axon radii ensembles (see Eq. (1)), the arithmetic mean radius (see Eq. (2)) and the effective radius (see Eq. (3) ) as $\hat{r}, \hat{\boldsymbol{r}}, \hat{r}_{\text {arith }}$, and $\hat{r}_{\text {eff }}$; corresponding reference values are denoted as $\tilde{r}, \tilde{\boldsymbol{r}}, \tilde{r}_{\text {arith }}$ and $\tilde{r}_{\text {eff }}$.

\subsection{Data acquisition}

Microscopy images from four human white matter samples of four different subjects were acquired: a corpus callosum (CC, male, 74 years, 24 hours postmortem delay, multiple tumors and multi organ failure), a corticospinal tract (CST, female, 89 years, 24 hours postmortem delay, heart failure), an optic chiasm (OC, male, 59 years, 48 hours postmortem delay, multi organ failure) and a sample obtained from the area dorsolateral of the olivary nucleus including the anterolateral system (AS, male, 81 years, 24 hours postmortem delay, multi organ failure). Following standard procedures, blocks were immersion-fixed in $3 \%$ paraformaldehyde and $1 \%$ glutaraldehyde in phosphate-buffered saline at $\mathrm{pH}$ 7.4. Then, smaller blocks of 1 to $4 \mathrm{~mm}$ edge length were cut, contrasted with osmium tetroxide and uranyl acetate, dehydrated in graded acetones, embedded in Durcupan resin and cut into semi- $(\sim 500 \mathrm{~nm})$ and ultra-thin $(\sim 50 \mathrm{~nm})$ sections. Semi-thin sections were stained with $1 \%$ toluidine blue.

In total, 13 lsLM images were acquired of semi-thin sections using a Zeiss AxioScan Z1 (objective: $40 \times$, numerical aperture: 0.95, resolution: $0.1112 \mu \mathrm{m} /$ pixel; resolution limit: $292 \mathrm{~nm}$ ) (see Table 1). For six regions of the CC sample, lsLM sections and matching EM sections were acquired, i.e., sections were cut within $100 \mu \mathrm{m}$ proximity (see Fig. 1). For the latter EM sections, images were acquired using a Zeiss LEO EM 912 Omega TEM at $80 \mathrm{kV}$ and digital micrographs were obtained with a dual-speed 2Kon-axis CCD camera-based YAG scintillator (TRS-Tröndle, resolution: $0.0043 \mu \mathrm{m} / \mathrm{pixel}$, resolution limit: $4 \mathrm{~nm})$. 
a)

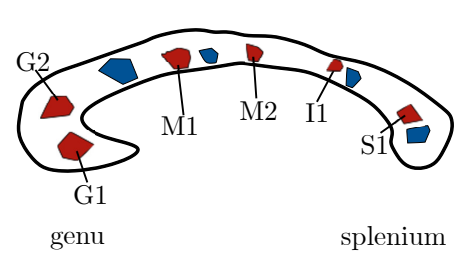

b)

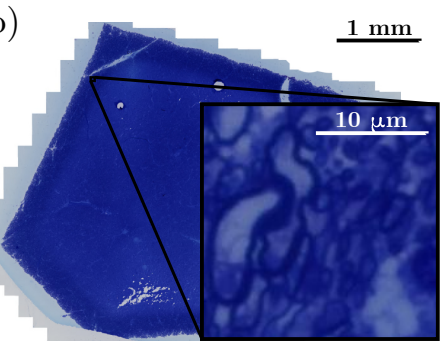

c)

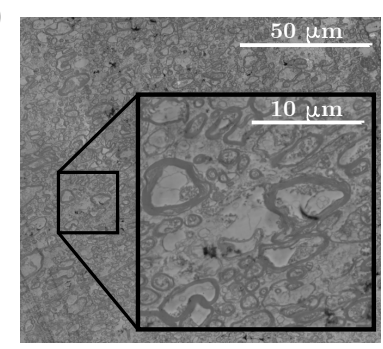

Figure 1: The human corpus callosum sample. The schematic of the sample (a) highlights the regions used for training (blue) and testing (red). For each region, one lsLM section was acquired. For the six test regions (red), matching lsLM and EM subsections were acquired: two sections from genu (G1, G2), two sections from midbody (M1, M2) and one section each from isthmus (I1) and splenium (S1). For section G1, the lsLM (b) and its matching EM (c) section are depicted as well as examples of subsections that were magnified to cover the same spatial extent $\left(20 \times 20 \mathrm{\mu m}^{2}\right)$ at common resolution.

\subsection{Data annotation}

\subsubsection{Training dataset}

For training of the CNN, we annotated 64 lsLM subsections of similar size $\left(70 \times 70 \mathrm{um}^{2}\right.$ to $120 \times 120 \mathrm{\mu m}^{2}$ ) originating from the training sections of the four tissue samples (see Table 1): 46 CC subsections, 4 OC subsections, 4 CST subsections and 10 AS subsections. To cover a wide range of appearance in axon shape and image contrast, some subsections were only partially annotated, i.e., pixels were assigned an ignore label and were not considered during training. As large axons were expected to have particular relevance for $r_{\text {eff }}$, but occur with low frequency, we assigned higher priority to the annotation of these axons.

\subsubsection{Test dataset}

We annotated subsections of the $N=6$ test regions (Fig. 1 1 ) as reference data to assess the error of $\hat{r}_{\text {arith }}$ (see Eq. (2p), the error of $\hat{r}_{\text {eff }}$ (see Eq. (3i) and the segmentation performance of the CNN in evaluation experiments (see Section 2.6). To account for the different characteristics of these measures (see Section 2.1), we generated separate reference subsections, which differ in the type of microscopy data (EM and lsLM), the subsection size and the annotation scope, i.e., selective and entire annotation of axons of particular size (see Table 2).

To assess $r_{\text {arith }}$, we annotated matching EM subsections $\left(S_{\mathrm{EM}}^{(n)}\right.$ with $n \in[1, N]$; see Table 2). Thereby, we generated references that accurately resolve even small $(r<$ $0.3 \mathrm{\mu m}$ ) axons below the resolution limit of lsLM, which we expected to have a relevant contribution towards the frequency-weighted $r_{\text {arith }}$.

As $r_{\text {eff }}$ is weighted towards the tail of the axon radii distribution (see Eq. (3)), accurate assessment requires sufficient sampling of the tail and thus subsections that cover large ensembles of axons $\left(S_{\text {lsLM,large }}^{(n, l)}\right.$ with $n \in[1, N]$ and $l \in\left[1, L_{\text {large }}\right]$; see Table 22. Because exhaustive annotation of these large ensembles was infeasible, we annotated only axons with a radius above a certain threshold $(r \geq 1.6 \mu \mathrm{m})$. This threshold was chosen so that $\hat{r}_{\text {eff }}$ was decreased by $50 \%$ when axons above this threshold were removed from the pooled axon radii ensemble of the corpus callosum lsLM sections evaluated with 
Table 2: The test dataset. For each of the $N=6$ test regions, high-resolution, large-scale light microscopy (lsLM) and electron microscopy (EM) subsections were annotated entirely or selectively (column: annotated axons). During evaluation experiments (see Section 2.6, these annotations were used partially or entirely (column: evaluated axons). Axon radii ranges used during evaluation experiments are as follows: small $(r<0.3 \mu \mathrm{m})$, medium-sized $(0.3 \mu \mathrm{m} \leq r<1.8 \mu \mathrm{m})$ and large $(r \geq 1.8 \mu \mathrm{m})$. $n \in[1, N]$ denotes the test region index. $l$ denotes the subsection index, which can be between 1 and the number subsections per region.

\begin{tabular}{|c|c|c|c|c|c|c|}
\hline $\begin{array}{l}\text { Subsection } \\
\text { symbol }\end{array}$ & Type & $\begin{array}{r}\text { Subsections } \\
\text { per region } n \\
\end{array}$ & $\begin{array}{l}\begin{array}{l}\text { Subsection } \\
\text { size }\left[\mathrm{mm}^{2}\right]\end{array} \\
\end{array}$ & $\begin{array}{l}\text { Annotated } \\
\text { axons }\end{array}$ & $\begin{array}{l}\text { Evaluated } \\
\text { axons }\end{array}$ & $\begin{array}{l}\text { Evaluation } \\
\text { experiment }\end{array}$ \\
\hline$S_{\mathrm{EM}}^{(n)}$ & EM & 1 & $50 \times 50$ & all & $\begin{array}{l}\text { all } \\
\text { small }\end{array}$ & $\begin{array}{l}\text { Error of } \hat{r}_{\text {arith }} \\
\text { Error of } \hat{r}_{\text {eff }}\end{array}$ \\
\hline$S_{\text {lsLM,large }}^{(n, l)}$ & $1 \mathrm{sLM}$ & $L_{\text {large }}=3$ & $350 \times 350$ & $\begin{array}{l}\text { axons with } \\
r \geq 1.6 \mu \mathrm{m} \\
\end{array}$ & $\begin{array}{l}\text { large } \\
\text { large } \\
\end{array}$ & $\begin{array}{l}\text { Segmentation performance } \\
\text { Error of } \hat{r}_{\text {eff }}\end{array}$ \\
\hline$S_{\mathrm{lsLM}}^{(n, l)}$ & lsLM & $L=5$ & $28 \times 28$ & all & $\begin{array}{l}\text { all } \\
\text { medium-sized }\end{array}$ & $\begin{array}{l}\text { Segmentation performance } \\
\text { Error of } \hat{r}_{\text {eff }}\end{array}$ \\
\hline
\end{tabular}

a prototype of the proposed method. However, for evaluation the threshold had to be adjusted to $1.8 \mu \mathrm{m}$ (for further explanation see Section 2.6.1). To complement the assessment of $\hat{r}_{\text {eff }}$ for the error introduced by smaller axons, we used the annotations of small axons in matching EM subsections $S_{\mathrm{EM}}$ and the annotations of remaining, mediumsized $(0.3 \mu \mathrm{m} \leq r<1.8 \mu \mathrm{m})$ axons in small lsLM subsections $\left(S_{\mathrm{lsLM}}^{(n, l)}\right.$ with $n \in[1, N]$ and $l \in[1, L]$; see Table 2]. The so-obtained split of the axon radii distribution for the assessment of $\hat{r}_{\text {eff }}$ into

(1) small axons $(r<0.3 \mu \mathrm{m})$,

(2) medium-sized axons $(0.3 \mu \mathrm{m} \leq r<1.8 \mu \mathrm{m})$ and

(3) large axons $(r \geq 1.8 \mu \mathrm{m})$

was used throughout different evaluation experiments to assess the contribution of individual axon radii ranges towards $\hat{r}_{\text {arith }}$ or $\hat{r}_{\text {eff }}$.

The segmentation performance of the CNN across the entire range of axon radii was assessed on small subsections $S_{\mathrm{lsLM}}^{(n, l)}$, whereas the segmentation performance for large axons on $S_{\text {lsLM,large }}^{(n, l)}$.

\subsubsection{Raters}

Annotation of lsLM subsections, i.e. both training and test subsections, was performed by a total of six raters (M. Morozova, B. Fricke, J.M. Oeschger, S. Papazoglou, T. Tabarin and L. Mordhorst). Each annotated subsection was crosschecked by a second rater. Initially, annotations were carried out in collaboration with two neuroanatomy experts (i.e., M. Morawski and M. Morozova) who were furthermore consulted in case of doubt. EM subsections were annotated by M. Morozova.

\subsection{Axon radius estimation pipeline}

Axon radius estimation was divided into three steps: semantic segmentation, instance segmentation and radius approximation (see Fig. 2). To perform semantic segmentation, i.e., to classify each pixel as either axon, myelin or background, we applied a CNN (see Section 2.5 in a sliding window manner (see Fig. 2a). To identify axon instances from individual pixels, we applied connected-component labeling (see Fig. 2b). Axon radii were 
a)

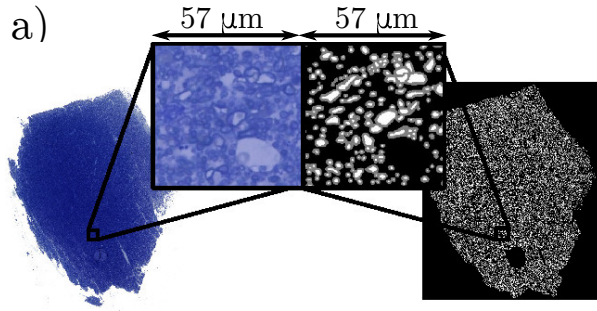

b)

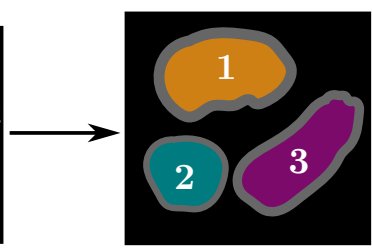

c)

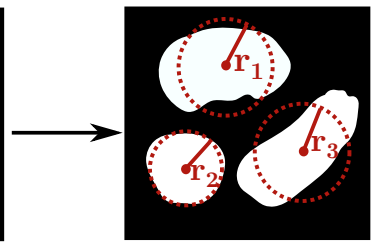

Figure 2: The axon radius estimation pipeline. (a) Pixel-wise classifications as axon, myelin or background were obtained through application of a semantic segmentation network, i.e. a U-Net [26] variant, in a sliding window manner. (b) Subsequently, axon instances were identified through connected component labeling of pixels that were classified as axon. (c) For each axon instance, the radius was estimated as the radius of a circle with equivalent area.

approximated as radii of circles with equivalent areas to those of the axon instances (short: circular equivalent) (see Fig. 22). Axon radii ensembles (see Eq. (1)) were composed from approximated radii. For annotated images, the semantic segmentation step was skipped.

\subsection{Semantic segmentation network}

Network Architecture. We used a CNN of the U-Net [26, 27] family with an input size of $512 \times 512$ pixels and four encoder stages. We employed transfer learning: the original encoders were replaced by EfficientNet-B3 28] encoders that were pretrained on the ImageNet dataset [29].

Training and Validation. The training process was split into pseudo-epochs of 150 random patches. Augmentations were employed on-the-fly during training: geometric, brightness and saturation transformations, Gaussian blurring and stain augmentation 30. Inputs were standardized with respect to the training dataset. We used stochastic gradient descent with Nesterov momentum (0.95), an initial learning rate of $10^{-2}$ and a learning rate decay of $\gamma=0.2$ every 50 epochs after initial 100 epochs to minimize a Lovàsz-softmax loss [31. All weights of the CNN were modified during training. We determined the optimal learning rate, $\gamma$ and the number of epochs in a 4 -fold crossvalidation with splits conducted at the level of entire subsections. The best model was determined in terms of the averaged dice score for axon and myelin and trained for 200 epochs. We used a framework [32] based on PyTorch [33] to carry out the training procedure.

\subsection{Evaluation of the performance of the radius estimation pipeline}

\subsubsection{Performance of the semantic segmentation network}

We considered the binary, pixel-wise classification task of discriminating between axon and background, i.e., all non-axon pixels were considered background, to evaluate balanced accuracy, dice, precision and recall. For all $N$ test regions, we evaluated all $L$ subsections with entirely annotated axons $\left(S_{\mathrm{lsLM}}^{(n, l)}\right.$ with $n \in[1, N]$ and $l \in[1, L]$; see Table 2 and all $L_{\text {large }}$ subsections selectively annotated for the assessment of large axons $\left(S_{\text {lsLM,large }}^{(n, l)}\right.$ with $n \in[1, N]$ and $l \in\left[1, L_{\text {large }}\right]$; see Table 2 . To compute the above 


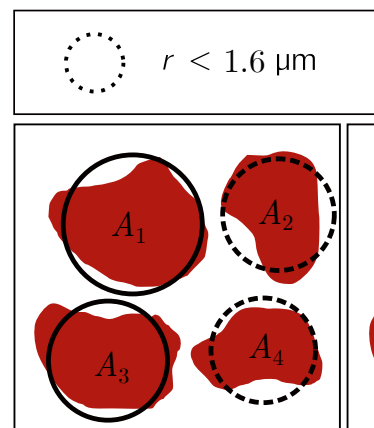

a) Annotation

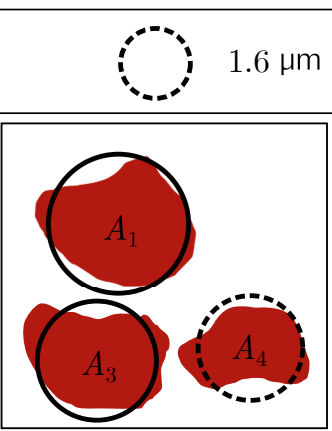

b) Reference
$6 \mu \mathrm{m} \leq r<1.8 \mu \mathrm{m}$

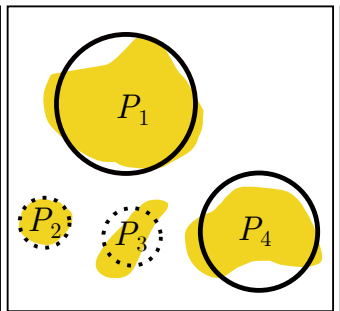

c) Prediction

\section{$r \geq 1.8 \mu \mathrm{m}$}

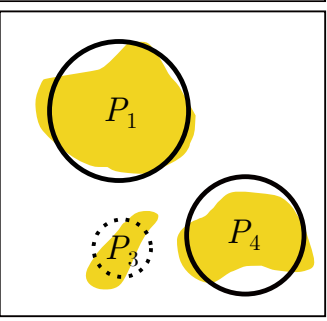

d) Large-axon prediction

Figure 3: Generation of reference and large-axon prediction for one subsection. The annotation (a) was filtered to generate the reference (b). Similarly, the prediction (c) was filtered to generate the large-axon prediction (d). For the reference (b), large $(r \geq 1.8 \mu \mathrm{m})$ axons were always considered (a$\mathrm{b}, A_{1}$ and $\left.A_{3}\right)$. Annotated axons with $1.6 \mu \mathrm{m} \leq r<1.8 \mu \mathrm{m}$ (a, $A_{2}$ and $\left.A_{4}\right)$ were used as follows: we assumed that predicted, large axons (e.g., in c-d, $\left.P_{4}\right)$ with no large, annotated counterpart but an annotated counterpart with $1.6 \mu \mathrm{m} \leq r<1.8 \mu \mathrm{m}$ were correctly detected, but marginally oversegmented. Therefore, we considered annotated axons with $1.6 \mu \mathrm{m} \leq r<1.8 \mu \mathrm{m}\left(\mathrm{a}-\mathrm{b}, A_{2}\right.$ and $\left.A_{4}\right)$ for the reference if a large, overlapping axon existed $\left(\mathrm{a}-\mathrm{b}, A_{4}\right)$ but discarded them otherwise $\left(\mathrm{a}, A_{2}\right)$. For the large-axon prediction $(\mathrm{d})$, axons were considered if they had $r>=1.8 \mu \mathrm{m}\left(\mathrm{c}-\mathrm{d}, P_{1}\right.$ and $\left.P_{4}\right)$ or if they had the highest pairwise dice similarity (c-d, $P_{1}, P_{3}$ and $\left.P_{4}\right)$ to a reference (b) axon. To compute the pairwise dice similarity, only the respective two axons were considered, whereas remaining pixels were considered background. Circles denote circular equivalent axon areas.

metrics for $S_{\mathrm{lsLM}}^{(n, l)}$, annotations served as an immediate reference for comparison with the prediction. To determine above metrics for $S_{\text {lsLM,large }}^{(n, l)}$, we filtered both annotation and prediction for large axons to generate reference and large-axon prediction (see Fig. 3. The corresponding aforementioned metrics were then computed using reference and largeaxon prediction. To summarize each metric over all subsections of all regions for $S_{\mathrm{lsLM}}^{(n, l)}$ and $S_{\text {lsLM,large }}^{(n, l)}$, we computed the mean of each metric, respectively.

\subsubsection{Error of $\hat{r}_{\text {arith }}$}

We assessed the error of $\hat{r}_{\text {arith }}$ by comparing estimates obtained from lsLM subsections $\left(S_{\text {lsLM,large }}^{(n, l)}\right.$ with $n \in[1, N]$ and $l \in\left[1, L_{\text {large }}\right]$; see Table 2 ) against references obtained from annotated EM subsections $\left(S_{\mathrm{EM}}^{(n)}\right.$; see Table 2). For $l$-th subsection of the $n$-th region, we computed an estimate $\hat{r}_{\text {arith }}^{(n, l)}$ from the predicted axon radii ensemble of $S_{\text {lsLM,large }}^{(n, l)}$ and a reference $\tilde{r}_{\text {arith }}^{(n, l)}$ from the EM-based axon radii ensemble $\tilde{\boldsymbol{r}}_{\mathrm{EM}}^{(n)}$ of the matching, annotated subsection $S_{\mathrm{EM}}^{(n)}$. Thereby, references were shared across all $L_{\text {large }}$ subsections per region, i.e., $\tilde{r}_{\text {arith }}^{(n, 1)}=\ldots=\tilde{r}_{\text {arith }}^{\left(n, L_{\text {large }}\right)}$. To assess accuracy and bias of $\hat{r}_{\text {arith }}^{(n, l)}$ with respect to $\tilde{r}_{\text {arith }}^{(n, l)}$ across all $N \cdot L_{\text {large }}$ subsections of all regions, we computed the normalized-root-meansquare error

$$
\mathrm{NRMSE}=\frac{\sqrt{\frac{1}{N \cdot L_{\text {large }}} \sum_{n=1}^{N} \sum_{l=1}^{L_{\text {large }}}\left(\hat{r}_{\text {arith }}^{(n, l)}-\tilde{r}_{\text {arith }}^{(n, l)}\right)^{2}}}{\frac{1}{N \cdot L_{\text {large }}} \sum_{n=1}^{N} \sum_{l=1}^{L_{\text {large }}} \tilde{r}_{\text {arith }}^{(n, l)}}
$$




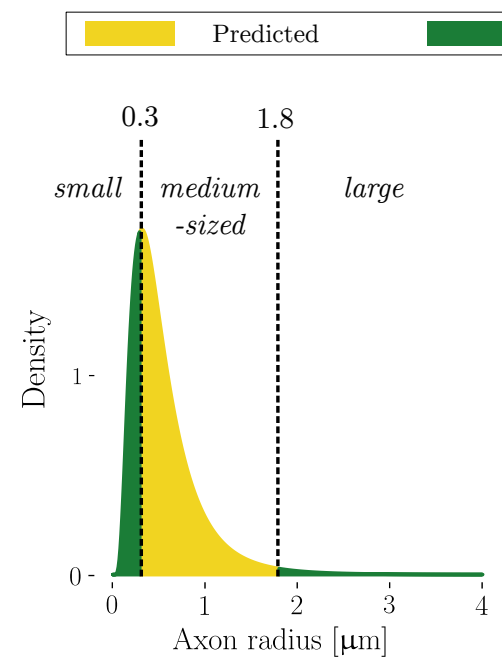

a) $\tilde{\boldsymbol{r}}^{(n, l)}$

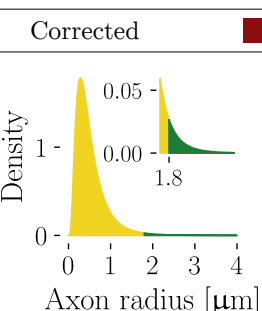

b) $\hat{\boldsymbol{r}}_{\text {small }}^{(n, l)}$

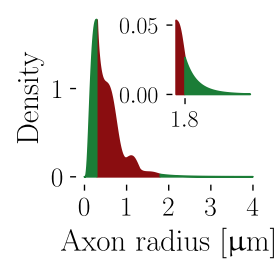

d) $\hat{\boldsymbol{r}}_{\text {medium }}^{(n, l, m)}$
Manipulated

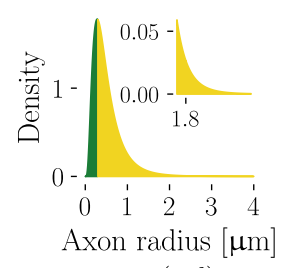

c) $\hat{\boldsymbol{r}}_{\text {large }}^{(n, l)}$

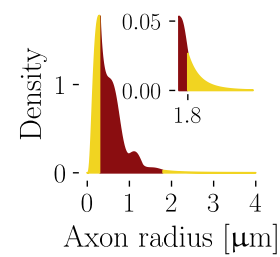

e) $\hat{\boldsymbol{r}}_{\text {all }}^{(n, l, m)}$

Figure 4: Generation of axon radii ensembles for the error assessment of $\hat{r}_{\text {eff }}$. For one subsection $(l$ of region $n$ ), the reference axon radii ensemble $\left(\tilde{\boldsymbol{r}}^{(n, l)}\right.$; a) was based on the predicted (yellow) axon radii ensemble $\left(\hat{\boldsymbol{r}}^{(n, l)}\right)$. Medium-sized axon radii remained unchanged. Small and large axon radii were corrected (green) from annotated data. To generate the erroneous axon radii ensembles (b-e), either corrections were not applied (b,c), axon radii were manipulated (red; d) or a combination of both was applied (e). Note, that multiple $(M=500)$ axon radii ensembles were generated to assess the error introduced in the medium-sized and entire axon radii range, of which $\hat{\boldsymbol{r}}_{\text {medium }}^{(n, l, m)}$ and $\hat{\boldsymbol{r}}_{\text {all }}^{(n, l, m)}$ denote the $m$-th $(m \in[1, M])$ axon radii ensemble, respectively.

and the normalized-mean-bias-error

$$
\mathrm{NMBE}=\frac{\sum_{n=1}^{N} \sum_{l=1}^{L_{\text {large }}} \hat{r}_{\text {arith }}^{(n, l)}-\tilde{r}_{\text {arith }}^{(n, l)}}{\sum_{n=1}^{N} \sum_{l=1}^{L_{\text {large }}} \tilde{r}_{\text {arith }}^{(n, l)}} .
$$

\subsubsection{Error of $\hat{r}_{\text {eff }}$}

The error of $\hat{r}_{\text {eff }}$ (see Eq. (3)) was assessed on large lsLM subsections $\left(S_{\text {lsLM,large }}^{(n, l)}\right.$ with $n \in[1, N]$ and $l \in\left[1, L_{\text {large }}\right]$; see Table 2). To distinctively assess the error due to axons in particular axon radii ranges (see Section 2.3.2), we compared axon radii ensembles with erroneous radii in the particular range against reference values.

For the $l$-th subsection of the $n$-th region, we generated a reference axon radii ensemble $\tilde{\boldsymbol{r}}^{(n, l)}$ and erroneous axon radii ensembles: one each for the small and large axon radii range $\left(\hat{\boldsymbol{r}}_{\text {small }}^{(n, l)}\right.$ and $\left.\hat{\boldsymbol{r}}_{\text {large }}^{(n, l)}\right)$; and multiple $(M=500)$ each for the medium-sized and entire axon radii range, of which $\hat{\boldsymbol{r}}_{\text {medium }}^{(n, l, m)}$ and $\hat{\boldsymbol{r}}_{\text {all }}^{(n, l, m)}$ denote the $m$-th $(m \in[1, M])$ erroneous axon radii ensemble (see Fig. 40. The generation of the aforementioned ensembles is detailed in the following paragraphs.

Generation of $\tilde{\boldsymbol{r}}^{(n, l)} . \tilde{\boldsymbol{r}}^{(n, l)}$ was generated by correcting the predicted axon radii ensemble $\hat{\boldsymbol{r}}^{(n, l)}$ obtained from $S_{\text {lsLM,large }}^{(n, l)}$ (see Table 2 in the range of small and large axon radii, whereas medium-sized axon radii remained unchanged (see Fig. 4a). 
Small axon radii of $\hat{\boldsymbol{r}}^{(n, l)}$ were corrected by substitution with the EM-based axon radii ensemble $\tilde{\boldsymbol{r}}_{\mathrm{EM}}^{(n)}$ obtained from the matching, annotated subsection $S_{\mathrm{EM}}^{(n)}$ (see Table 2]. As $\tilde{\boldsymbol{r}}_{\mathrm{EM}}^{(n)}$ was smaller than $\hat{\boldsymbol{r}}^{(n, l)}$, direct substitution was inappropriate. Instead, we discarded small axons of $\hat{\boldsymbol{r}}^{(n, l)}$ and randomly sampled small axons from $\tilde{\boldsymbol{r}}_{\mathrm{EM}}^{(n)}$ with replacement until the ratio of small and remaining axons equaled the ratio observed in $\tilde{\boldsymbol{r}}_{\mathrm{EM}}^{(n)}$.

Large axon radii of $\hat{\boldsymbol{r}}^{(n, l)}$ were corrected by substitution with the axon radii ensemble $\tilde{\boldsymbol{r}}_{\text {lsLM,large }}^{(n, l)}$ obtained from annotations on $S_{\text {lsLM,large }}^{(n, l)}$ (see Table 2). To replace under- or oversegmented axons in $\hat{\boldsymbol{r}}^{(n, l)}$ and to complement $\hat{\boldsymbol{r}}^{(n, l)}$ by falsely missed axons, we added axon radii of $\tilde{\boldsymbol{r}}_{\text {lsLM,large }}^{(n, l)}$. If existent, we replaced corresponding axon radii of $\hat{\boldsymbol{r}}^{(n, l)}$ with a counterpart from $\tilde{\boldsymbol{r}}_{\text {lsLM,large }}^{(n, l)}$. To determine a corresponding axon in $\hat{\boldsymbol{r}}^{(n, l)}$ for each axon in $\tilde{\boldsymbol{r}}_{\text {lsLM,large }}^{(n, l)}$, we computed the pairwise dice similarity as explained in Fig. 3 . Remaining, large axon radii of $\hat{\boldsymbol{r}}^{(n, l)}$ were considered falsely detected and were discarded for $\tilde{\boldsymbol{r}}^{(n, l)}$.

Generation of $\hat{\boldsymbol{r}}_{\text {small }}^{(n, l)} . \hat{\boldsymbol{r}}_{\text {small }}^{(n, l)}$ was generated analogously to $\tilde{\boldsymbol{r}}^{(n, l)}$ except that small axons were not corrected (see Fig. $4 \mathrm{p}$ ). To estimate an upper bound of the error introduced by missing small axons, we repeated the experiment after discarding all small axons of $\hat{\boldsymbol{r}}_{\text {small }}^{(n, l)}$.

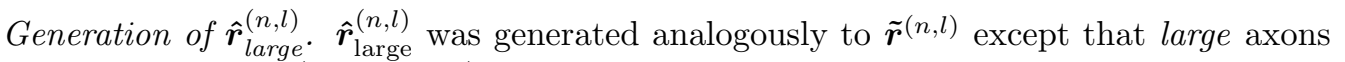
were not corrected (see Fig. 4 .).

Generation of $\hat{\boldsymbol{r}}_{\text {medium }}^{(n, l, m)}$. Due to the lack of annotated data for correction of mediumsized axons, we effectively used medium-sized axon radii of $\hat{\boldsymbol{r}}^{(n, l)}$ as a reference, i.e., $\tilde{\boldsymbol{r}}^{(n, l)} \approx \hat{\boldsymbol{r}}^{(n, l)}$ for medium-sized axons (see Fig. 4h). Erroneous, medium-sized axon radii were generated by manipulating $\tilde{\boldsymbol{r}}^{(n, l)}$ according to an error model in a Monte Carlo simulation (see Fig. 5). As a consequence of employing a Monte Carlo simulation approach, we did not generate one, but $M=500$ erroneous axon radii ensembles. The parameters of the error model used in the Monte Carlo simulation were obtained from corresponding predictions and references (here: annotations) for the set of $N \cdot L=30$ subsections $P=\left\{S_{\mathrm{lsLM}}^{(1,1)}, \ldots, S_{\mathrm{lsLM}}^{(1, L)}, \ldots, S_{\mathrm{lsLM}}^{(N, 1)}, \ldots S_{\mathrm{lsLM}}^{(N, L)}\right\}$ (see Table 2).

To generate the $m$-th erroneous axon radii ensemble $\hat{\boldsymbol{r}}_{\text {medium }}^{(n, l, m)}, \tilde{\boldsymbol{r}}^{(n, l)}$ was manipulated as follows: first, randomly drawn, missed (false negative; FN) axons were removed; second, axon radii of correctly detected (true positive; TP) axons were perturbed; third, randomly drawn, falsely detected (false positive; FP) axons were added. The following paragraphs explain for the $m$-th iteration how model parameters were determined and how the so-obtained model was applied to generate $\hat{\boldsymbol{r}}_{\text {medium }}^{(n, l, m)}$. For brevity, we omit subsection, region and simulation iteration indices $(l, n$ and $m)$.

To determine the parameters of the error model, we pooled over five pairs of corresponding predictions and references for subsections randomly drawn from the aforementioned $P$ (see Fig. 5k.1 and Fig. 5k.2). The error was modeled as a function of the axon radius. For this, we binned reference axons according to their radii into $J=5$ bins, chosen to contain the same number of axons (see Fig. 5 . .2 , bottom). For each bin $j \in[1, J]$, corresponding predictions and references were compared to classify non-overlapping axons as FP or FN and fully or partially overlapping axons as TP (see Fig. 55.3). Then, 
we counted the number of FN $\left(N_{\mathrm{FN}, j}\right), \mathrm{FP}\left(N_{\mathrm{FP}, j}\right)$ and $\mathrm{TP}\left(N_{\mathrm{TP}, j}\right)$ axons. Finally, we determined the false negative rate

$$
\mathrm{FNR}_{j}=\left(N_{\mathrm{FN}, j}\right) /\left(N_{\mathrm{FN}, j}+N_{\mathrm{TP}, j}\right),
$$

the residuals

$$
\Delta r_{j}=\hat{r}_{j, i}-\tilde{r}_{j, i}
$$

between predicted $\left(\hat{r}_{j, i}\right.$ with $\left.i \in\left[1, N_{\mathrm{TP}, j}\right]\right)$ and reference $\left(\tilde{r}_{j, i}\right.$ with $\left.i \in\left[1, N_{\mathrm{TP}, j}\right]\right)$ radii of TP axons, the false discovery rate

$$
\mathrm{FDR}_{j}=\left(N_{\mathrm{FP}, j}\right) /\left(N_{\mathrm{FP}, j}+N_{\mathrm{TP}, j}\right)
$$

and the distribution of FP axon radii $\left(r_{\mathrm{FP}, \mathrm{j}}\right)$ (see Fig. 5f.4).

To apply the error model for bin $j$, the original axon radii ensemble with $N_{j}$ axons in bin $j$ was manipulated in three steps: First, $\hat{N}_{\mathrm{FN}, j}\left(=\mathrm{FNR}_{j} \cdot N_{j}\right)$ randomly drawn FN axons were removed (see Fig. 5 b.1). Then, an error ( $\epsilon_{k}$ with $\left.k \in\left[1, N_{j}-\hat{N}_{\mathrm{FN}, j}\right]\right)$ sampled from $\Delta r_{j}$ was added to each axon radius (see Fig. 5b.2). Finally, $\hat{N}_{\mathrm{FP}, j}\left(=\mathrm{FPR}_{j} \cdot N_{j}\right)$ axons were added from the distribution of $\mathrm{FP}$ axon radii $\left(r_{\mathrm{FP}, \mathrm{j}}\right)$ to obtain the erroneous axon radii ensemble (see Fig. 5b.3 and see Fig. 5d).

Generation of $\hat{\boldsymbol{r}}_{\text {all }}^{(n, l, m)} . \hat{\boldsymbol{r}}_{\text {all }}^{(n, l, m)}$ was generated analogously to $\hat{\boldsymbol{r}}_{\text {medium }}^{(n, l, m)}$ but with the following difference: instead of manipulating $\tilde{\boldsymbol{r}}^{(n, l)}$, we manipulated $\hat{\boldsymbol{r}}^{(n, l)}$ (see Fig. 44).

From reference and erroneous axon radii ensembles, we computed effective axon radii $\tilde{r}_{\text {eff }}^{(n, l)}, \hat{r}_{\text {eff,small }}^{(n, l)}, \hat{r}_{\text {eff,large }}^{(n, l)} \hat{r}_{\text {eff,medium }}^{(n, l, m)}$ and $\hat{r}_{\text {eff,all }}^{(n, l, m)}$ according to Eq. 33 for all $L_{\text {large }}$ subsections of all $N$ regions and all $M$ simulation iterations. For $\hat{r}_{\text {eff,small }}^{(n, l)}$ and $\hat{r}_{\text {eff,large }}^{(n, l)}$, accuracy and bias with respect to $\tilde{r}_{\text {eff }}^{(n, l)}$ were assessed across all $N \cdot L_{\text {large }}$ subsections of all regions in terms of NRMSE and NMBE (defined analogously to Eqs. (4) and (5)). For $\hat{r}_{\text {eff,medium }}^{(n, l, m)}$ and $\hat{r}_{\text {eff,all }}^{(n, l, m)}, \mathrm{NRMSE}_{m}$ and $\mathrm{NMBE}_{m}$ with respect to $\tilde{r}_{\text {eff }}^{(n, l)}$ were computed per iteration $m$ and summarized by their mean and standard deviation.

\subsubsection{Sensitivity of $\hat{r}_{\text {arith }}$ and $\hat{r}_{\text {eff }}$ to variation of the image intensity}

We assessed whether the influence of spatially varying intensity, e.g. introduced by staining heterogeneity, affected the capability of our method to map anatomy-related, spatial variation of $\hat{r}_{\text {arith }}$ and $\hat{r}_{\text {eff }}$ across whole lsLM sections. For qualitative analysis, we generated spatially smoothed maps of $\hat{r}_{\text {arith }}$ and $\hat{r}_{\text {eff }}$ by computing the average of randomly positioned subsections (area: $0.12 \mathrm{\mu m}^{2}$ ) and visually compared the patterns of the spatially smoothed maps to those of the corresponding lsLM images. For quantitative analysis, maps of $\hat{r}_{\text {arith }}, \hat{r}_{\text {eff }}$ and the image intensity were generated similar to those above but sampled on an equally spaced grid (grid pixel area: $0.12 \mu \mathrm{m}^{2}$ ). To obtain a scalar value for the image intensity, we applied gray scale conversion. Then, grid pixels of sections with similar axon radii distribution (G1, G2, M1, M2) were pooled and the correlation between image intensity and mapped radii was computed. As visual inspection suggested that small axons were particularly difficult to resolve in strongly 
bioRxiv preprint doi: https://doi.org/10.1101/2021.06.03.446739; this version posted June 3, 2021. The copyright holder for this preprint (which was not certified by peer review) is the author/funder, who has granted bioRxiv a license to display the preprint in perpetuity. It is made available under aCC-BY 4.0 International license.

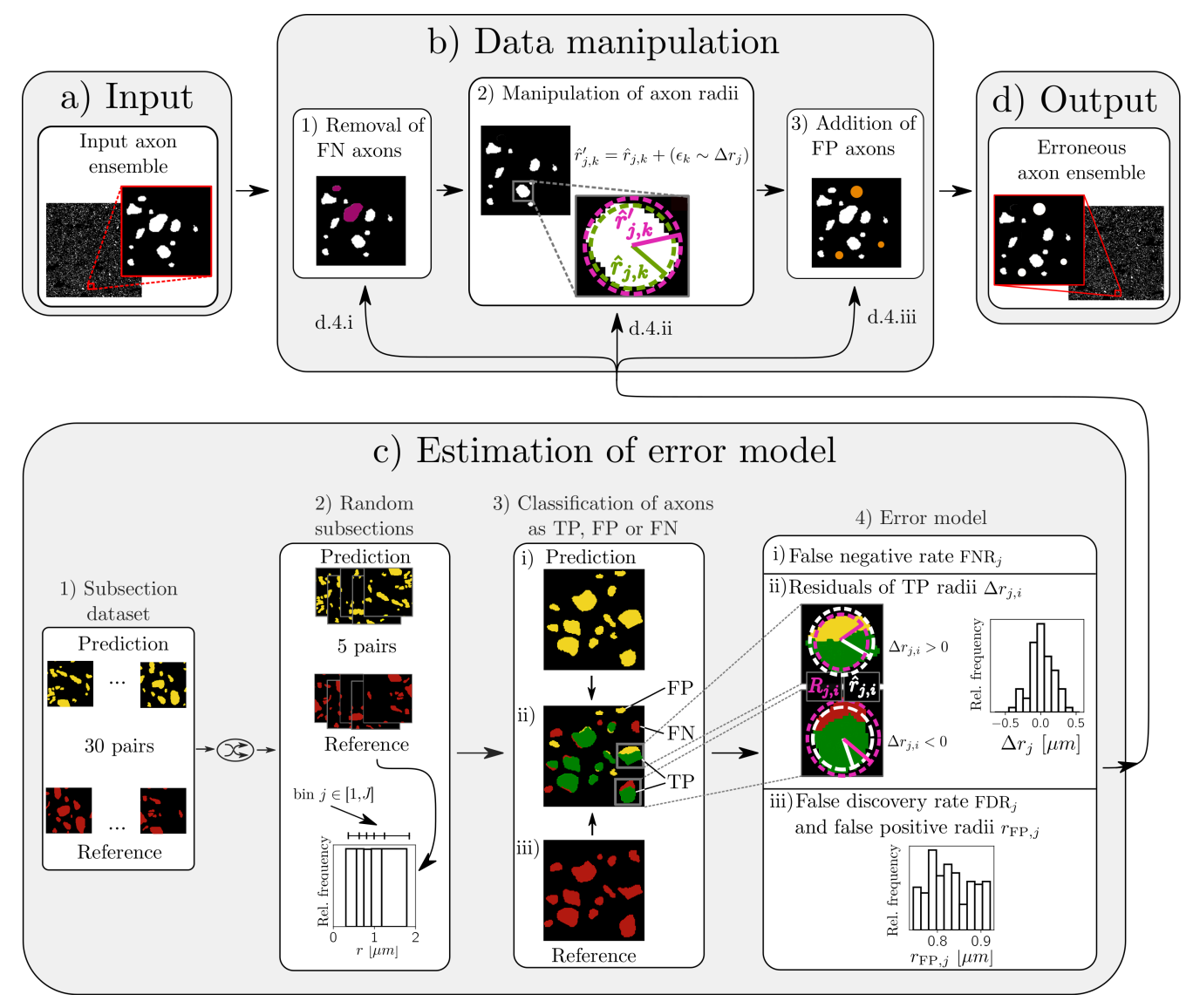

Figure 5: Schematic of the generation of axon ensembles with erroneous, medium-sized axon radii. The input axon ensemble (a) was manipulated (b) based on an error model (c) to generate axon radii ensembles with erroneous medium-sized axon radii (d). To model the error as a function of the axon radius, distinct parameters of the error model were determined per axon radii bin $j \in[1, J=5]$ (see details on the binning below). To obtain an erroneous axon radii ensemble (d), we employed the error model (c.4) for each bin $j$ as follows: First, randomly drawn, missed (false negative; FN) axon radii were removed (b.1, purple) according to the false negative rate $\left(\mathrm{FNR}_{j}\right.$; c.4.i, see Eq. (6)). Then, axon radii were perturbed (b.2) according to the residuals of partially or fully detected (true positive; TP) axons $\left(\Delta r_{j}\right.$; c.4.ii, see Eq. 7 ). Finally, randomly drawn, falsely detected (false positive; FP) axon radii were added (b.3, orange) according to the false discovery rate $\left(\mathrm{FDR}_{j}\right.$; c.4.iii, see Eq. (8)) and the distribution of FP axon radii $\left(r_{\mathrm{FP}, j}\right.$; c.4.iii). To determine the parameters of the error model, we pooled over five pairs of corresponding predictions (c.2, yellow) and references (c.2, red) randomly drawn from 30 pairs (c.1). The $J$ bins were chosen to contain the same number of reference axon radii per bin. For each bin $j$, axons of corresponding predictions (c.3.i) and references (c.3.iii) were compared (c.3.ii) to classify non-overlapping axons as FP (entirely yellow in c.3.ii) or FN (entirely red in c.3.ii) and partially or fully overlapping axons as TP (partially or fully green in c.3.ii and c.4.ii). Then (c.4), we determined the parameters of the error model. 
Table 3: Segmentation metrics. Each metric was computed as the mean over all annotated subsections for all axons and only large axons $\left(S_{\mathrm{lsLM}}^{(n, l)}\right.$ and $S_{\mathrm{lsLM}, \text { large }}^{(n, l)}$; see Table 2 .

\begin{tabular}{lrr}
\hline Metric & All axons & Large axons \\
\hline Balanced accuracy & 0.85 & 0.94 \\
Dice & 0.77 & 0.89 \\
Precision & 0.82 & 0.90 \\
Recall & 0.74 & 0.89 \\
\hline
\end{tabular}

stained areas, the above experiments were performed with and without considering small axons to test this hypothesis.

\subsubsection{Sensitivity of $r_{\text {eff }}$ to outstandingly large axons}

To evaluate how much $r_{\text {eff }}$ is affected by outstandingly large axons, we investigated how $r_{\text {eff }}$ changed as a function of a varying threshold $\tau$ when only axons with $r<\tau$ were considered for the computation of $r_{\text {eff. }} \tau$ was chosen to cover the whole range of observed axon radii for a given ensemble. In particular, we assessed the worst case in which the largest individual axon was missed. To exclude estimation errors from this experiment, we considered only reference data, i.e., the reference axon ensembles generated in Section 2.6.3. Furthermore, to carry out this analysis at a scale as close as possible to the cross-sectional size of typical voxels of a human MRI system $\left(1 \mathrm{~mm}^{2}\right.$ or larger), we computed $\tilde{r}_{\text {eff }}$ from combined axon radii ensembles for which we pooled over all $L_{\text {large }}$ subsections per test region, yielding $\tilde{\boldsymbol{r}}_{\text {pooled }}^{(n)}=\bigcup_{l=1}^{L_{\text {large }}} \tilde{\boldsymbol{r}}^{(n, l)}$ for the $n$-th test region. Thereby, we obtained $\tilde{r}_{\text {eff }}$ from the largest axon ensembles available for each test region based on combined areas of about $0.37 \mathrm{~mm}^{2}$.

\section{Results}

\subsection{Performance of the semantic segmentation network}

The segmentation metrics (balanced accuracy, dice, precision and recall, see Table 3) are given for all axons and only large axons. All metrics were higher when only large axons were evaluated than when all axons were evaluated.

\subsection{Error of $\hat{r}_{\text {arith }}$}

$\hat{r}_{\text {arith }}$ deviated from the line of unity, overestimating $\tilde{r}_{\text {arith }}$ by $16 \%$ (NMBE) and yielding an NRMSE of $21.5 \%$ (see Fig. 6).

\subsection{Error of $\hat{r}_{\text {eff }}$}

The overall accuracy and bias as assessed by NRMSE and NMBE were $7.2 \% \pm 0.5 \%$ and $-1.7 \% \pm 0.4 \%$ (see Fig. $7 \mathrm{~d}$ ). The agreement between $\hat{r}_{\text {eff }}$ and $\tilde{r}_{\text {eff }}$ in the small (NRMSE: $0.6 \%$ and NMBE: $0.4 \%$ ) and medium-sized (NRMSE: $1.9 \% \pm 0.3 \%$ and NMBE: $0.5 \% \pm 0.4 \%$ ) axon radii range was higher than in the large (NRMSE: $7.4 \%$ and NMBE: $-2.7 \%$ ) axon radii range (see Fig. $7 \mathrm{~A}-\mathrm{c}$ ). The error in the large axon radii range translated into the range of all axons (see Fig. 75-d). The error due to small axons increased when small axons were discarded for the estimation of $\hat{r}_{\text {eff,small }}$ (NRMSE: $1.5 \%$ and NMBE: $1.5 \%$ ) (see Fig. 7a). 


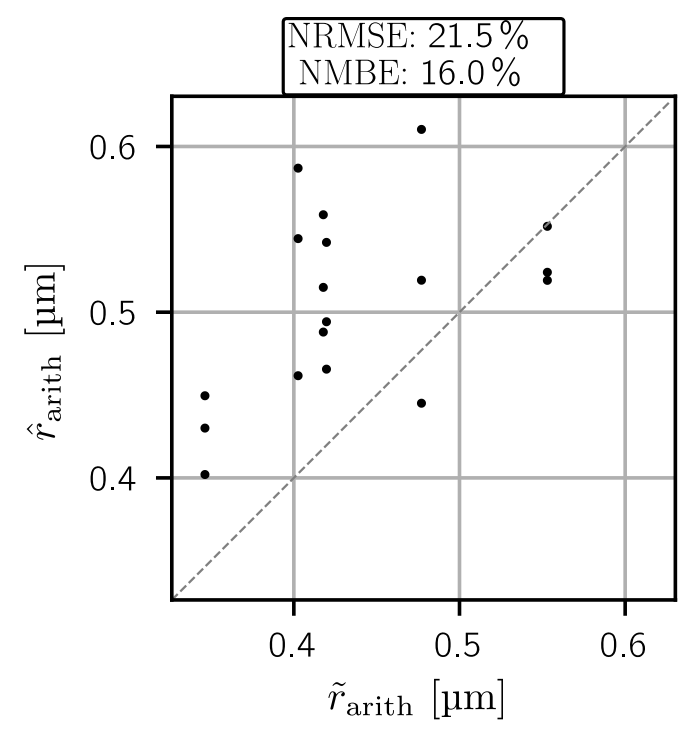

Figure 6: Error of $\hat{r}_{\text {arith. }}$ Each point compares an lsLM-based estimate $\left(\hat{r}_{\text {arith }}\right)$ against its EM-based reference $\left(\tilde{r}_{\text {arith }}\right)$. The dashed line represents the line of unity. NRMSE and NMBE over all subsections were $21.5 \%$ and $16 \%$.

\subsection{Sensitivity of $\hat{r}_{\text {arith }}$ and $\hat{r}_{\text {eff }}$ to variation of the image intensity}

The spatial variation of $r_{\text {arith }}$ resembled the image intensity distribution of the corresponding lsLM section (see Fig. 8p, top row and Fig. 8b). In contrast, maps of $\hat{r}_{\text {eff }}$ had a high local heterogeneity, which was not observed in the image intensity distribution of the corresponding lsLM section (see Fig. 8 $\mathrm{a}$, bottom row and Fig. 8 8 ). These observations were supported by a strong correlation $\left(\rho=0.80, p<10^{-5}\right)$ between $\hat{r}_{\text {arith }}$ and the image intensity, which was reduced when small axons were discarded $(\rho=0.50$, $p<10^{-5}$ ) (see Fig. 8c, top left and top right). In contrast, $r_{\text {eff }}$ did not show a significant correlation with the image intensity (see Fig. 88, bottom row).

\subsection{Sensitivity of $r_{\text {eff }}$ to outstandingly large axons}

$\tilde{r}_{\text {eff }}$ increased nonlinearly as a function of $\tau$ but with decreasing slope (see Fig. 9 9 ). For large $\tau$, the lines fragment due to the sparse occurrence of large axons. Compared to other regions, the influence of the largest axon on $\tilde{r}_{\text {eff }}$ was particularly strong in region M2: $\tilde{r}_{\text {eff }}$ decreased by $14.5 \%$ when the largest axon was discarded (see Fig. 9 $\mathrm{a}$ ). The largest axon was much larger other axons across all regions and its elongated shape suggested that this axon was oriented almost parallel to the cutting plane, i.e., its axon radius was strongly overestimated by the circular equivalent approximation (see Fig. 9p). The influence of the largest axon was smaller for the remaining regions: $\tilde{r}_{\text {eff }}$ decreased by $0.9 \%$ to $3 \%$ when discarding the largest axon (see Fig. 9 a).

\section{Discussion}

We investigated the potential of CNN-based segmentation on high-resolution, largescale light microscopy (lsLM) sections to narrow the scale gap between histological ref- 
bioRxiv preprint doi: https://doi.org/10.1101/2021.06.03.446739; this version posted June 3, 2021. The copyright holder for this preprint (which was not certified by peer review) is the author/funder, who has granted bioRxiv a license to display the preprint in perpetuity. It is made available under aCC-BY 4.0 International license.
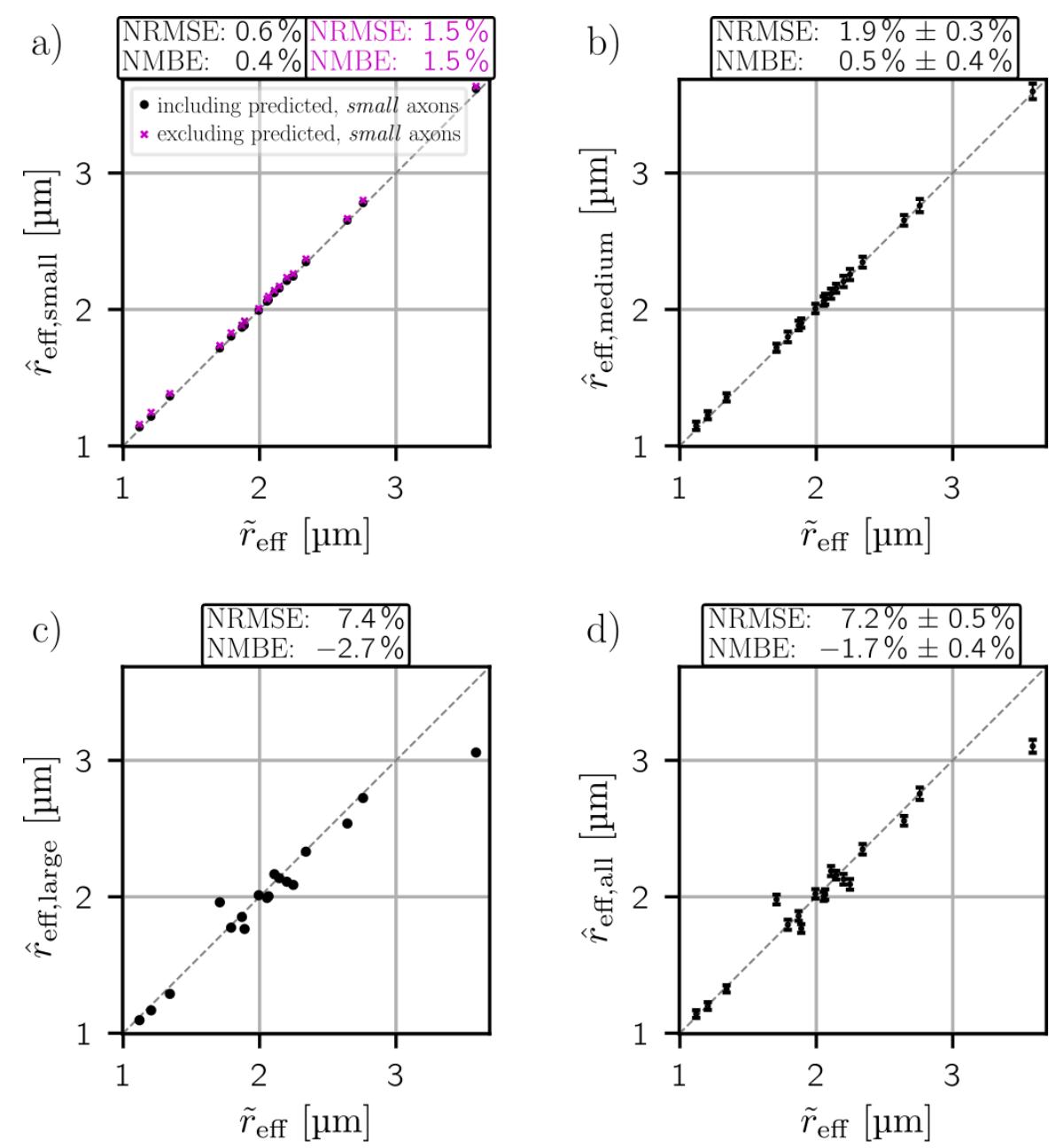

Figure 7: Error of $\hat{r}_{\text {eff }}$. Depicted are comparisons of estimates $\left(\hat{r}_{\text {eff }}\right)$ against a common reference $\left(\tilde{r}_{\text {eff }}\right)$, taking errors introduced in different axon radii ranges into account, i.e., small $\left(\hat{r}_{\text {eff,small }} ; r<0.3 \mu \mathrm{m}\right)(\mathrm{a})$, medium-sized $\left(\hat{r}_{\text {eff,medium }} ; 0.3 \mu \mathrm{m} \leq r<1.8 \mu \mathrm{m}\right)(\mathrm{b})$, large $\left(\hat{r}_{\text {eff,large }} ; r \geq 1.8 \mu \mathrm{m}\right)(\mathrm{c})$ and all $\left(\hat{r}_{\text {eff,all }}\right)$ axons (d). Each point corresponds to a distinct lsLM subsection. The dashed line represents the line of unity. Two cases were considered for the error introduced in the small axon range: estimation of $\hat{r}_{\text {eff,small }}$ with (magenta) and without (black) including predicted, small axons. Error bars for results derived through Monte Carlo simulation (b, d) denote mean and standard deviation per subsection. NRMSE and NMBE were computed over all subsections according to Eqs. (4) and (5). Mean and standard deviation of NRMSE and NMBE across Monte Carlo simulation iterations are given, if applicable (b, d). 
bioRxiv preprint doi: https://doi.org/10.1101/2021.06.03.446739; this version posted June 3, 2021. The copyright holder for this preprint (which was not certified by peer review) is the author/funder, who has granted bioRxiv a license to display the preprint in perpetuity. It is made available under aCC-BY 4.0 International license.

a)

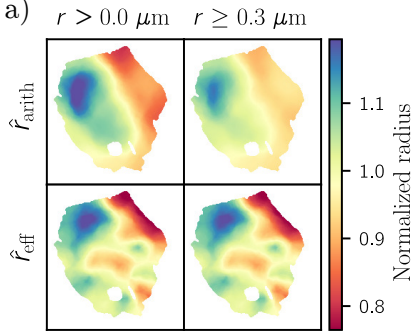

b)

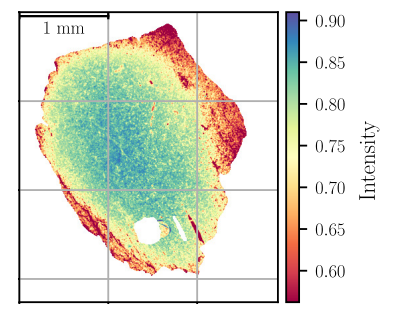

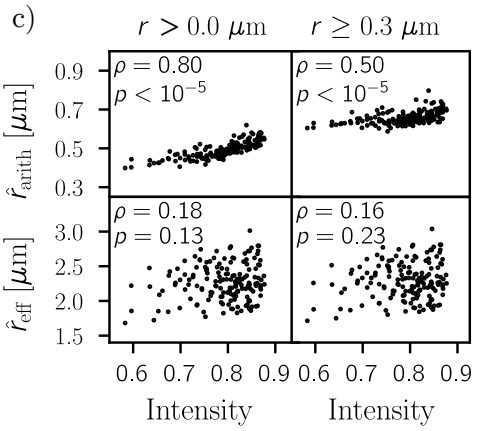

Figure 8: Sensitivity of $\hat{r}_{\text {arith }}$ and $\hat{r}_{\text {eff }}$ to variation of the image intensity. Depicted are: spatially smoothed maps of $\hat{r}_{\text {arith }}$ and $\hat{r}_{\text {eff }}$ (a), the lsLM image of section M1 (b) adjusted to illustrate the correlation with maps of $\hat{r}_{\text {arith }}\left(\right.$ a), and scatter plots between ensemble mean axon radii $\left(\hat{r}_{\text {arith }}\right.$ and $\left.\hat{r}_{\text {eff }}\right)$ and lsLM image intensities (c). The correlation plots (c) pool across four sections (G1, G2, M1, M2). The $p$-values have been multiplied by the number of sections to correct for multiple comparisons ( $\rho$ is the correlation coefficient).

a)

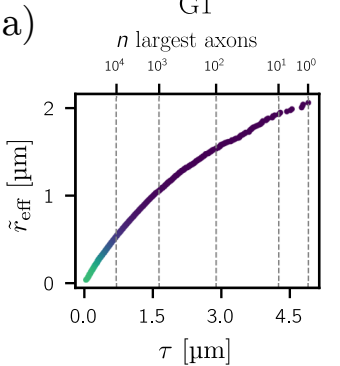

G2

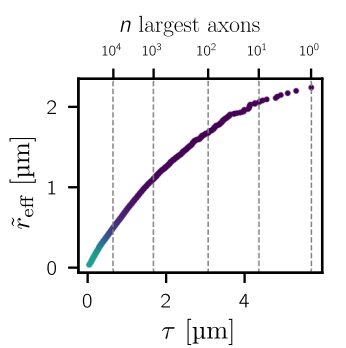

M1

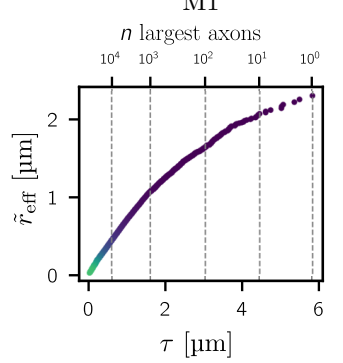

$\mathrm{M} 2$

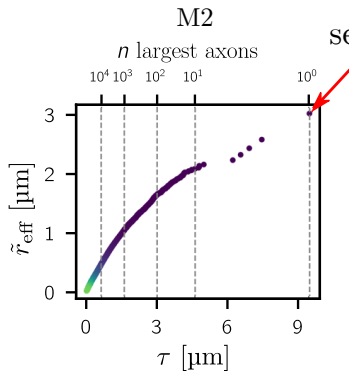

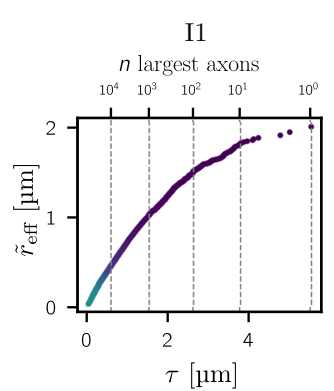

S1

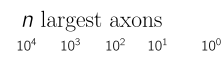

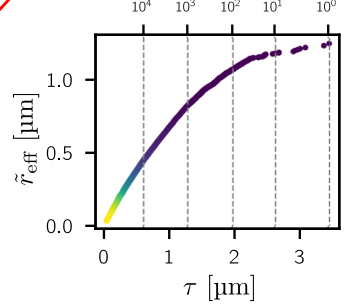

$n$ largest axons $1 \cdot 10^{4} \quad 3 \cdot 10^{4} \quad 5 \cdot 10^{4}$

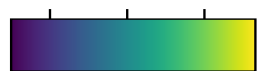

b)

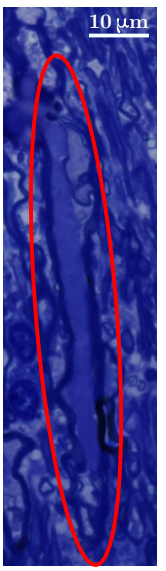

Figure 9: Sensitivity of $r_{\text {eff }}$ to outstandingly large axons. Depicted are the values of $\tilde{r}_{\text {eff }}$ for the six test regions of the corpus callosum sample (a) when considering only axons with radius of $r<\tau$ for the computation of $\tilde{r}_{\text {eff }}$. The marker colors indicate the order of axons sorted by their radius in descending order according to the colorbar in the top right. For orientation, powers of 10 are marked on top of the plots (a). The extracted lsLM subsection (b) shows the largest axon $(r=9.46 \mu \mathrm{m})$ observed across all regions, causing the jump of $\tilde{r}_{\text {eff }}$ for region M2 between largest and second-largest axon. The elongated shape of this axon is likely due to the axon being oriented almost parallel to the cutting plane of the two-dimensional section. When discarding this axon, $\tilde{r}_{\text {eff }}$ decreased from $3.02 \mu \mathrm{m}$ to $2.58 \mu \mathrm{m}$, i.e., a decrease of $14.5 \%$. For the remaining regions, the decrease of $\tilde{r}_{\text {eff }}$ ranged from $0.9 \%$ to $3 \%$. The total number of axons ranged from $3 \cdot 10^{4}$ to $6.3 \cdot 10^{4}$. 
erence data and MRI voxels for the validation of MRI-based radius estimation in human brain tissue. The proposed pipeline accurately estimates the effective axon radius $\left(r_{\text {eff }}\right)$ in a human corpus callosum on sections spanning several cross-sections of typical voxels of human MRI systems ( $1 \mathrm{~mm}^{2}$ or larger) and is thus a promising candidate for the validation of MRI-based radius estimation in the human brain. However, the arithmetic mean radius $\left(r_{\text {arith }}\right)$, which is commonly reported in neuroanatomical studies, is less accurately estimated.

\section{Error of $r_{\text {arith }}$ and $r_{\text {eff }}$}

To assess the estimation error of $r_{\text {eff }}$ representatively for cross-sections of MRI voxels ( $1 \mathrm{~mm}^{2}$ or larger) of a human MRI system, sufficient sampling of the tail of the axon radii distribution is required. Therefore, we investigated large ensembles of axons including at least 10,000 axons per sample. To address the challenge of assessing the error of $r_{\text {eff }}$ on large ensembles of axons, we divided the axon radii distribution into three ranges and employed distinct annotation and evaluation approaches. Thereby, we circumvented exhaustive annotation of smaller axons and enabled complete annotation of large $(r \geq$ $1.8 \mathrm{\mu m}$ ) axons, which we expected to be of greatest relevance for $r_{\text {eff }}$.

Across the entire range of axon radii, we conclude higher suitability to estimate $r_{\text {eff }}$ than $r_{\text {arith }}$ due to higher accuracy (normalized-root-mean-square-error: $7.2 \%$ vs. $21.5 \%$ ) and lower bias (normalized-mean-bias-error: $-1.7 \%$ vs. $16 \%$ ). Assessment of individual ranges revealed that erroneous, large axons predominantly determine the estimation accuracy of $r_{\text {eff }}$. In contrast, erroneous, small axons $(r<0.3 \mu \mathrm{m})$ below the resolution limit of lsLM introduced only a minor overestimation, even when they were neglected altogether for estimating $r_{\text {eff. }}$. Thus, the potential of lsLM to sample the tail of the axon radii distribution in large field-of-views outweighs its limited capability to resolve small axons for mapping $r_{\text {eff }}$.

While we assessed the presented pipeline with particular focus on the ensemble mean radii of segmented axons, i.e., $r_{\text {arith }}$ and $r_{\text {eff }}$, we employed pixel-wise optimization during training. The metrics commonly used to evaluate pixel-wise segmentation reflect the better suitability to estimate $r_{\text {eff }}$ : large axons, that determine $r_{\text {eff }}$, were best segmented.

\section{Mapping anatomy-related, spatial variation across whole sections}

Toluidine staining introduces low-frequency variation of the image intensity across whole lsLM sections. In a spatial correlation analysis, we identified this variation as a confounding factor for mapping $r_{\text {arith }}$ but not for mapping $r_{\text {eff }}$. In the light of moderate errors, spatial variation of $r_{\text {eff }}$ seems anatomy-related. As $r_{\text {arith }}$ was particularly confounded when small axons were taken into account, small axons seem particularly prone to staining effects. The inaccurate resolution of small axons may explain the observed overestimation of $r_{\text {arith }}$. For $r_{\text {eff }}$, the correlation with the image intensity was hardly affected by inclusion or rejection of small axons which underlines their minor contribution towards $r_{\text {eff. }}$

\section{Sensitivity of $r_{\text {eff }}$ to outstandingly large axons}

Due to the tail-weighting of $r_{\text {eff }}$, individual, outstandingly large axons may strongly contribute towards $r_{\text {eff }}$ and thus strongly decrease estimation accuracy in case of erroneous segmentation. We assessed this potential source of error by discarding the largest axon for the computation of $r_{\text {eff }}$ in axon ensembles of at least 30,000 axons. 
The strongest contribution (14.5\% in region M2) of an individual axon was due to an outlier. Across the remaining regions, the contribution was smaller (0.9\% to $3 \%)$, but still notable, considering that these axons represented only $0.002 \%$ to $0.003 \%$ of the axon ensembles. For the outlier-region M2, the largest axon $(\mathrm{r}=9.46 \mu \mathrm{m})$ was oriented almost parallel to the cutting plane, resulting in an elongated shape. Thus, circular equivalent approximation may largely overestimate axon radii and bias the estimation of $r_{\text {eff. }}$ Instead, axon radii may be estimated based on the minor axes of ellipsoids fitted to the axon areas.

The investigated lsLM subsections (area: $\sim 0.37 \mathrm{~mm}^{2}$ ) were smaller than the crosssection of a typical MRI voxel $\left(1 \mathrm{~mm}^{2}\right.$ or larger). In the latter, we expect reduced potential of individual axons to bias $r_{\text {eff }}$ due to the larger axon ensemble size.

\section{Limitations and future directions}

Although the method accurately estimated $r_{\text {eff }}$ for different axon radii ensembles sampled across the corpus callosum, further investigation is required to assess how well the model generalizes and how well the overall method translates to other brain areas.

Recent, automated methods for large-scale axon segmentation use different acquisition techniques 24, 25, and segmentation algorithms 24, 25, which, however, were trained on perfusion-fixed brain tissue of mice or rats. Future studies can use our method as a benchmark to validate the aforementioned approaches for $r_{\text {eff }}$ estimation in human brain tissue.

To assess $r_{\text {arith }}$, we compared lsLM-based estimates against EM-based references from close-by cut sections. This choice of reference has two limitations: first, the EM-based axon ensembles were smaller, i.e., covering only 5 to $10 \%$ of their lsLM-based counterparts; second, spatial misalignment arised from section-to-section distance and unknown in-section location. However, we assumed that representative estimation of the frequencyweighted $r_{\text {arith }}$ is rather enabled by accurate resolution of frequently occurring axons than by large ensemble size or exact spatial alignment. Consequently, we regarded EM as a more suitable reference than lsLM because EM can resolve all frequently occurring axons, including small axons below the resolution limit of lsLM. Indeed, we found small axons to be particularly prone to variation of the image intensity in lsLM which in turn led to consistent overestimation of $r_{\text {arith }}$. The overestimation of $r_{\text {arith }}$ indicates that a bias of the method predominantly determined the inaccuracy of $r_{\text {arith }}$ rather than inappropriate reference data.

The annotation of microscopy slides is prone to errors and inter-observer variability, in particular in the presence of staining and tissue degradation due to the immersion-fixation used in this study. Employing strategies that address noisy and uncertain annotations, e.g. by design of specific loss functions may improve axon segmentation accuracy 34 and thus radius estimation accuracy.

\section{Conclusion}

The presented pipeline accurately maps the MRI-visible, effective radius $\left(r_{\text {eff }}\right)$ in a human corpus callosum sample by combining high-resolution, large-scale light microscopy (lsLM) with deep learning at the cross-sectional scale of typical MRI voxels $\left(1 \mathrm{~mm}^{3}\right.$ or larger). Since the pipeline is based on the fast, cheap and simple to perform lsLM 
measurement, it can easily be used beyond the realm of MRI-based radius models, e.g., to generate a representative, neuroanatomical atlas of the ensemble of large axons across the human corpus callosum. Generalization to other brain areas with different axon radii ensembles is yet to be demonstrated.

\section{Code and data availability}

The source code and data used in this study will be made publicly available upon publication.

\section{Ethics}

For samples used in this study, the entire procedure of case recruitment, acquisition of the patient's personal data, the protocols and the informed consent forms, performing the autopsy and handling the autopsy material have been approved by the responsible authorities (Approval \#205/17-ek).

\section{CRediT authorship contribution statement}

Laurin Mordhorst: Conceptualization, Formal Analysis, Investigation, Methodology, Software, Visualization, Writing - Original Draft. Maria Morozova: Investigation, Writing - Review \& Editing: Sebastian Papazoglou: Investigation, Supervision, Writing - Review \& Editing. Björn Fricke: Investigation. Jan Malte Oeschger: Investigation, Writing - Review \& Editing. Thibault Tabarin: Conceptualization, Investigation. Henriette Rusch: Investigation, Writing - Review \& Editing. Carsten Jäger: Resources, Writing - Review \& Editing. Stefan Geyer: Funding Acquisition. Nikolaus Weiskopf: Funding Acquisition, Writing - Review \& Editing. Markus Morawski: Funding Acquisition, Resources, Writing - Review \& Editing. Siawoosh Mohammadi: Conceptualization, Funding Acquisition, Methodology, Writing - Review \& Editing, Supervision.

\section{Acknowledgements}

The research leading to these results has received funding from the European Research Council under the European Union's Seventh Framework Programme (FP7/2007-2013) / ERC grant agreement $n^{\circ} 616905$.

This work was supported by the German Research Foundation (DFG Priority Program 2041 "Computational Connectomics", [MO 2397/5-1; MO 2249/3-1; GE 2967/1-1], by the Emmy Noether Stipend: MO 2397/4-1) and by the BMBF (01EW1711A and B) in the framework of ERA-NET NEURON and the Forschungszentrums Medizintechnik Hamburg (fmthh; grant 01fmthh2017).

We are grateful to Dr. René Werner and Amra Hot for insightful discussions. 


\section{Conflict of interest}

The Max Planck Institute for Human Cognitive and Brain Sciences has an institutional research agreement with Siemens Healthcare. NW was a speaker at an event organized by Siemens Healthcare and was reimbursed for the travel expenses.

\section{References}

[1] J. Veraart, D. Nunes, U. Rudrapatna, E. Fieremans, D. K. Jones, D. S. Novikov, N. Shemesh, Noninvasive quantification of axon radii using diffusion MRI, eLife 9 (2020) e49855. doi:10.7554/ eLife.49855

[2] D. C. Alexander, P. L. Hubbard, M. G. Hall, E. A. Moore, M. Ptito, G. J. M. Parker, T. B. Dyrby, Orientationally invariant indices of axon diameter and density from diffusion MRI, NeuroImage 52 (4) (2010) 1374-1389. doi:10.1016/j.neuroimage.2010.05.043.

[3] Y. Assaf, T. Blumenfeld-Katzir, Y. Yovel, P. J. Basser, AxCaliber: A Method for Measuring Axon Diameter Distribution from Diffusion MRI, Magn Reson Med 59 (6) (2008) 1347-1354. doi:10. $1002 / \mathrm{mrm} .21577$

[4] M. Andersson, H. M. Kjer, J. Rafael-Patino, A. Pacureanu, B. Pakkenberg, J.-P. Thiran, M. Ptito, M. Bech, A. Bjorholm Dahl, V. Andersen Dahl, T. B. Dyrby, Axon morphology is modulated by the local environment and impacts the noninvasive investigation of its structure-function relationship, Proc Natl Acad Sci USA 117 (52) (2020) 33649-33659. doi:10.1073/pnas.2012533117.

[5] H. Schmidt, T. R. Knösche, Action potential propagation and synchronisation in myelinated axons, PLOS Computational Biology 15 (10) (2019) e1007004. doi:10.1371/journal.pcbi.1007004.

[6] M. Drakesmith, R. Harms, S. U. Rudrapatna, G. D. Parker, C. J. Evans, D. K. Jones, Estimating axon conduction velocity in vivo from microstructural MRI, NeuroImage 203 (2019) 116186. doi: 10.1016/j.neuroimage.2019.116186

[7] S. G. Waxman, Determinants of conduction velocity in myelinated nerve fibers, Muscle \& Nerve 3 (2) (1980) 141-150. doi:10.1002/mus.880030207

[8] D. C. Alexander, T. B. Dyrby, M. Nilsson, H. Zhang, Imaging brain microstructure with diffusion MRI: Practicality and applications, NMR Biomed 32 (4) (2019) e3841. doi:10.1002/nbm.3841.

[9] N. Stikov, J. S. W. Campbell, T. Stroh, M. Lavelée, S. Frey, J. Novek, S. Nuara, M.-K. Ho, B. J. Bedell, R. F. Dougherty, I. R. Leppert, M. Boudreau, S. Narayanan, T. Duval, J. Cohen-Adad, P.-A. Picard, A. Gasecka, D. Côté, G. B. Pike, In vivo histology of the myelin g-ratio with magnetic resonance imaging, Neuroimage 118 (2015) 397-405. doi:10.1016/j.neuroimage.2015.05.023

[10] N. Weiskopf, S. Mohammadi, A. Lutti, M. F. Callaghan, Advances in MRI-based computational neuroanatomy: From morphometry to in-vivo histology, Curr Opin Neurol 28 (4) (2015) 313-322. doi:10.1097/WC0.0000000000000222

[11] N. Weiskopf, L. Edwards, G. Helms, S. Mohammadi, E. Kirilina, Quantitative magnetic resonance imaging of brain anatomy and in-vivo histology, Nat. Rev. Phys.

[12] L. M. Burcaw, E. Fieremans, D. S. Novikov, Mesoscopic structure of neuronal tracts from timedependent diffusion, NeuroImage 114 (2015) 18-37. doi:10.1016/j.neuroimage.2015.03.061

[13] F. Sepehrband, D. C. Alexander, N. D. Kurniawan, D. C. Reutens, Z. Yang, Towards higher sensitivity and stability of axon diameter estimation with diffusion-weighted MRI, NMR Biomed 29 (3) (2016) 293-308. doi:10.1002/nbm.3462

[14] L. Leenen, J. Meek, R. Nieuwenhuys, Unmyelinated fibers in the pyramidal tract of the rat: A new view, Brain Research 246 (2) (1982) 297-301. doi:10.1016/0006-8993(82)91179-9.

[15] M. A. Biedenbach, J. L. DeVito, A. C. Brown, Pyramidal tract of the cat: Axon size and morphology, Exp Brain Res 61 (2) (1986) 303-310. doi:10.1007/BF00239520

[16] M. Nilsson, S. Lasič, I. Drobnjak, D. Topgaard, C.-F. Westin, Resolution limit of cylinder diameter estimation by diffusion MRI: The impact of gradient waveform and orientation dispersion, NMR in Biomedicine 30 (7) (2017) e3711. doi:10.1002/nbm.3711

[17] F. Aboitiz, A. B. Scheibel, R. S. Fisher, E. Zaidel, Fiber composition of the human corpus callosum, Brain Research 598 (1) (1992) 143-153. doi:10.1016/0006-8993(92)90178-C

[18] D. Graf von Keyserlingk, U. Schramm, Diameter of axons and thickness of myelin sheaths of the pyramidal tract fibres in the adult human medullary pyramid, Anat Anz 157 (2) (1984) 97-111.

[19] R. Caminiti, H. Ghaziri, R. Galuske, P. R. Hof, G. M. Innocenti, Evolution amplified processing with temporally dispersed slow neuronal connectivity in primates, Proc. Natl. Acad. Sci. U.S.A. 106 (46) (2009) 19551-19556. doi:10.1073/pnas.0907655106 
[20] D. Liewald, R. Miller, N. Logothetis, H.-J. Wagner, A. Schüz, Distribution of axon diameters in cortical white matter: An electron-microscopic study on three human brains and a macaque, Biol Cybern 108 (5) (2014) 541-557. doi:10.1007/s00422-014-0626-2

[21] A. Horowitz, D. Barazany, I. Tavor, M. Bernstein, G. Yovel, Y. Assaf, In vivo correlation between axon diameter and conduction velocity in the human brain, Brain Struct Funct 220 (3) (2015) 1777-1788. doi:10.1007/s00429-014-0871-0

[22] G. M. Innocenti, R. Caminiti, F. Aboitiz, Comments on the paper by Horowitz et al. (2014), Brain Struct Funct 220 (3) (2015) 1789-1790. doi:10.1007/s00429-014-0974-7

[23] L. Mordhorst, M. Morozova, S. Papazoglou, B. Fricke, J. M. Oeschger, H. Rusch, C. Jäger, M. Morawski, N. Weiskopf, S. Mohammadi, Human Axon Radii Estimation at MRI Scale, in: Proceedings of the 2021 German Workshop on Medical Image Computing, Springer, Wiesbaden, 2021, pp. 180-185. doi:10.1007/978-3-658-33198-6_45

[24] A. Zaimi, M. Wabartha, V. Herman, P.-L. Antonsanti, C. S. Perone, J. Cohen-Adad, AxonDeepSeg. Automatic axon and myelin segmentation from microscopy data using convolutional neural networks, Sci Rep 8 (1) (2018) 3816. doi:10.1038/s41598-018-22181-4

[25] A. Abdollahzadeh, I. Belevich, E. Jokitalo, A. Sierra, J. Tohka, DeepACSON automated segmentation of white matter in 3D electron microscopy, Communications Biology 4 (1) (2021) 1-14. doi: $10.1038 / \mathrm{s} 42003-021-01699-\mathrm{w}$

[26] O. Ronneberger, P. Fischer, T. Brox, U-Net: Convolutional Networks for Biomedical Image Segmentation, in: N. Navab, J. Hornegger, W. M. Wells, A. F. Frangi (Eds.), Medical Image Computing and Computer-Assisted Intervention - MICCAI 2015, Lecture Notes in Computer Science, Springer International Publishing, Cham, 2015, pp. 234-241. doi:10.1007/978-3-319-24574-4_28

[27] P. Yakubovskiy, Segmentation_models.pytorch (2020). URL https://github.com/qubvel/segmentation_models.pytorch

[28] M. Tan, Q. Le, EfficientNet: Rethinking Model Scaling for Convolutional Neural Networks, in: International Conference on Machine Learning, PMLR, 2019, pp. 6105-6114.

[29] J. Deng, W. Dong, R. Socher, L. Li, Kai Li, Li Fei-Fei, ImageNet: A large-scale hierarchical image database, in: 2009 IEEE Conference on Computer Vision and Pattern Recognition, 2009, pp. 248255. doi:10.1109/CVPR.2009.5206848.

[30] M. Macenko, M. Niethammer, J. S. Marron, D. Borland, J. T. Woosley, Xiaojun Guan, C. Schmitt, N. E. Thomas, A method for normalizing histology slides for quantitative analysis, in: 2009 IEEE International Symposium on Biomedical Imaging: From Nano to Macro, IEEE, Boston, MA, USA, 2009, pp. 1107-1110. doi:10.1109/ISBI.2009.5193250

[31] M. Berman, A. R. Triki, M. B. Blaschko, The Lovász-Softmax Loss: A Tractable Surrogate for the Optimization of the Intersection-Over-Union Measure in Neural Networks, in: Proceedings of the IEEE Conference on Computer Vision and Pattern Recognition, 2018, pp. 4413-4421.

[32] W. A. et al. Falcon, et al., PyTorch Lightning (2019). URL https://github.com/PyTorchLightning/pytorch-lightning

[33] A. Paszke, S. Gross, F. Massa, A. Lerer, J. Bradbury, G. Chanan, T. Killeen, Z. Lin, N. Gimelshein, L. Antiga, A. Desmaison, A. Kopf, E. Yang, Z. DeVito, M. Raison, A. Tejani, S. Chilamkurthy, B. Steiner, L. Fang, J. Bai, S. Chintala, PyTorch: An Imperative Style, High-Performance Deep Learning Library, Advances in Neural Information Processing Systems 32.

[34] D. Karimi, H. Dou, S. K. Warfield, A. Gholipour, Deep learning with noisy labels: Exploring techniques and remedies in medical image analysis, Medical Image Analysis 65 (2020) 101759. doi:10.1016/j.media.2020.101759 